\title{
Rendimiento diagnóstico del marcador tumoral CA 19-9 en la diferenciación entre patología bilio-pancreática benigna y maligna*
}

\author{
Drs. JAIME JANS B. ${ }^{1,2}$, MARÍA J. TALMA R. ${ }^{2}$, MARIO ALMONACID R. ${ }^{2}$, JAVIER CRUZ M. ${ }^{1,2}$, \\ MAURICIO CÁCERES P. ${ }^{1,2}$, CARLA ROSENFELD M. ${ }^{3}$, GLADYS JARA O. ${ }^{4}$ \\ 1 Servicio de Cirugía, Hospital Base de Osorno. \\ 2 Campo Clínico Osorno, Facultad de Medicina, Universidad Austral de Chile. \\ 3 Instituto de Medicina Preventiva Veterinaria, Facultad de Ciencias Veterinarias. Universidad Austral de Chile. \\ 4 Servicio de Laboratorio Clínico, Hospital Base de Osorno. \\ Osorno, Chile.
}

\begin{abstract}
Accuracy of the tumor marker CA 19-9 in the differentiation of benign and malignant bilio-pancreatic diseases

Introduction: CA 19-9 has been identified as a derivative of sialic Lewis blood group A and is expressed in $95 \%$ of the population. Several studies have documented an overproduction of CA 19-9 in malignant pancreatic and biliary tree diseases. The objective of this study is to determine the accuracy of the tumor marker CA 19-9 differentiating benign and malignant bilio-pancreatic diseases. Material and Methods: Diagnostic test study. We reviewed the records of all patients with malignant bilio-pancreatic diseases and benign biliary calculous diseases evaluated in Hospital Base Osorno between August 2007 and December 2011, with CA 19-9 as part of their study. Results: 71 patients met the inclusion criteria, 17 men and 54 women, with a mean age of $60.7 \pm 15.3$ years old. Twenty nine (40.8\%) cases were benign and $42(59.2 \%)$ cases malignant. For a cutoff level of $37 \mathrm{U} / \mathrm{ml}$ the sensitivity, specificity, positive predictive value (PPV) and negative predictive value (NPV) to differentiate benign from malignant disease was $81 \%, 72.4 \%, 81 \%$ and $72.4 \%$, respectively. For a cut off level of $130 \mathrm{U} / \mathrm{ml}$ specificity and PPV increased to $96.6 \%$ and $96.4 \%$, respectively. Conclusions: The use of CA 19-9 is useful in the diagnosis of patients with suspected bilio-pancreatic malignant disease. The optimization of the normal published value can help to improve accuracy.

Key words: Tumor markers, Ca 19-9, pancreatic cancer, cholangiocarcinoma, choledocholithiasis, cholecystitis, neoplasms, bilio-pancreatic disease, biliary calculous disease.
\end{abstract}

\section{Resumen}

Introducción: El CA 19-9 se ha identificado como un derivado siálico del grupo sanguíneo Lewis A y se expresa en el $95 \%$ de la población. Numerosos estudios han documentado una sobreproducción de CA

*Recibido el 12 de noviembre de 2012 y aceptado para publicación el 17 de diciembre de 2012.

Los autores no refieren conflictos de interés.

Correspondencia: Dr. Jaime Jans B.

Av. Guillermo Bühler 1712, Osorno, Chile.

jaimejans@gmail.com 
19-9 en tumores malignos del árbol biliar y páncreas. El objetivo de este estudio es determinar la utilidad del marcador tumoral CA 19-9 en la diferenciación de patología bilio-pancreática benigna y maligna. Material y Métodos: Estudio de pruebas diagnósticas. Se revisaron los antecedentes de todos los pacientes con patología bilio-pancreática maligna y enfermedad litiásica biliar benigna, evaluados en el Hospital Base de Osorno entre agosto de 2007 y diciembre de 2011, a los que se les haya solicitado CA 19-9 como parte de su estudio. Resultados: 71 pacientes cumplieron los criterios de inclusión, 17 hombres y 54 mujeres, con una media de $60,7 \pm 15,3$ años de edad. Veintinueve $(40,8 \%)$ casos correspondieron patología benigna y $42(59,2 \%)$ casos a patología maligna. Para un valor de corte de $37 \mathrm{U} / \mathrm{ml}$ la sensibilidad, especificidad, valor predictivo positivo (VPP) y valor predictivo negativo (VPN) para diferenciar enfermedad benigna de maligna fue de $81 \%, 72,4 \%, 81 \%$ y $72,4 \%$, respectivamente. Para un valor de corte de $130 \mathrm{U} /$ $\mathrm{ml}$ la especificidad y el VPP aumentaron a 96,6\% y 96,4\%, respectivamente. Conclusiones: El uso del Ca 19-9 es útil en el proceso diagnóstico de pacientes con sospecha de patología bilio-pancreática maligna. La optimización de los valores sobre el valor de normalidad publicado puede ayudar a mejorar su rendimiento.

Palabras clave: Marcadores tumorales, Ca 19-9, cáncer de páncreas, colangiocarcinoma, coledocolitiasis, colecistitis, neoplasias, enfermedad bilio-pancreática, enfermedad biliar litiásica.

\section{Introducción}

Los marcadores tumorales son sustancias producidas o inducidas por la célula neoplásica que reflejan su crecimiento y/o actividad y que permiten conocer la presencia, evolución o respuesta terapéutica de un tumor maligno. La mayoría de ellos no son específicos de un tumor ${ }^{1}$. El CA 19-9 (antígeno carbohidrato) se ha identificado como un derivado siálico del grupo sanguíneo Lewis A y se expresa en el $95 \%$ de la población. Se consideran normales las concentraciones inferiores a $37 \mathrm{U} / \mathrm{ml}$. Numerosos estudios han documentado una sobreproducción de CA 19-9 en tumores malignos de páncreas ${ }^{2-4} \mathrm{y}$ del árbol biliar ${ }^{5,6}$. También se ha visto una sobreproducción en otras neoplasias malignas (estómago, hígado, colon y recto) $)^{7,8}$ y patologías benignas bilio-pancreáticas, como pancreatitis, colangitis y coledocolitiasis ${ }^{9,10}$.

El rendimiento en la diferenciación de enfermedades bilio-pancreáticas benignas y malignas para un valor de corte de $37 \mathrm{U} / \mathrm{ml}$ se ha reportado bajo, con sensibilidad y especificidad que no superan el $86 \%$ y el $70 \%$, respectivamente, por lo que se ha tratado de establecer valores de corte que permitan mejorar su rendimiento, lo que ha resultado en un aumento de la especificidad y el valor predictivo positivo $^{11,12}$.

La disponibilidad de recursos imagenológicos es aún limitada en el sistema público del país, por lo que contar con un marcador que nos permita diferenciar entre patología benigna y maligna seria importante para permitir una priorización en el estudio y tratamiento. El objetivo de este estudio es determinar la utilidad del marcador tumoral CA 19-9 como herramienta diagnóstica en la diferenciación de patología bilio-pancreática benigna y maligna en nuestro centro.

\section{Material y Método}

\section{Diseño}

Estudio de pruebas diagnósticas.

\section{Pacientes (criterios de inclusión)}

Se revisaron los antecedentes de todos los pacientes con patología bilio-pancreática maligna (cáncer vesicular, colangiocarcinoma, hepatocarcinoma, adenocarcinoma de páncreas y ampolla de Vater) y enfermedad litiásica biliar benigna (colecistitis crónica, colecistitis aguda, coledocolitiasis con y sin colangitis) evaluados en el Hospital Base de Osorno entre agosto de 2007 y diciembre de 2011, a los que se les haya solicitado CA 19-9 (Reactivo: Ca 19-9 SIEMENS; Equipo: ADVIA Centaur XP SIEMENS; Método: Quimioluminiscencia directa) como parte de su estudio. Se definió como enfermedad maligna aquellos casos con confirmación histopatológica de cáncer y aquellos casos sin diagnóstico histopatológico de cáncer, pero con estudio imagenológico categórico de tumores avanzados locoregionalmente y/o con imágenes de enfermedad metastásica, que hayan fallecido con cuadro clínico compatible luego de un seguimiento en la unidad de cuidados paliativos. Se definió enfermedad benigna aquellos casos con confirmación histopatológica de benignidad y casos de coledocolitiasis con o sin colangitis diagnosticados por colangiografía directa endoscópica o intraoperatoria.

\section{Fuentes}

Los datos se recolectaron de los registros de fichas clínicas, del Servicio de Anatomía Patológica, del Laboratorio Clínico y del Registro Civil.

\section{Variables}

Las variables consideradas fueron: edad, sexo, diagnóstico, CA 19-9, recuento de leucocitos, PCR, 
bilirrubina total y fosfatasas alcalinas (tomados al inicio del proceso diagnóstico y anterior a cualquier estudio histológico), estudio imagenológico (ecografía abdominal, tomografía computada (TC) de abdomen y pelvis y colangioresonancia magnética $(\mathrm{CRM}))$ y estudio histopatológico.

\section{Análisis estadístico}

Para el análisis estadístico se utilizó el programa Statgraphics Plus 5.1. Se realizó estadística descriptiva, así como el análisis de regresión y correlación para variables cuantitativas. Para comparar los grupos se realizó pruebas de hipótesis paramétricas (t-student) y no paramétricas (Mann-Whitney) según la determinación de normalidad a través de la prueba de Shapiro Wilk, y para variables dicotómicas se utilizó la prueba de $\chi^{2}$. Se consideró estadísticamente significativo un nivel de confianza del $95 \%$ con un valor $\mathrm{p}<0,05$. Además se calculó la sensibilidad, especificidad, VPP y VPN de la prueba diagnóstica CA 19-9. Se construyó una curva ROC para determinar el valor de corte óptimo del CA 19-9 y con este valor se calculó la sensibilidad, especificidad, VPP y VPN de la prueba. Para evaluar estadísticamente el área bajo la curva se aplicó la prueba de suma de rangos de Wilcoxon y se determinó significancia estadística un valor $>0,5$; fuera del intervalo de confianza.

\section{Resultados}

En el período estudiado, a 71 pacientes se les solicitó el marcador tumoral CA 19-9 como parte de su estudio, por tener ecografía sospechosa de neoplasia maligna (vesicular, biliar o periampular), ictericia obstructiva o ambos. La distribución por géneros fue de 17 hombres y 54 mujeres, con una media de edad de $60,7 \pm 15,3$ años (rango 20-88). Veintinueve casos $(40,8 \%)$ correspondieron a patología benigna y 42 casos $(59,2 \%)$ a patología maligna. Sesenta y cuatro pacientes $(90,1 \%)$ fueron estudiados con ecografía abdominal, 38 pacientes $(53,5 \%)$ con TC de abdomen y pelvis, y 2 pacientes $(2,8 \%)$ con CRM. En los casos de patología benigna el diagnóstico fue establecido con biopsia en 20 casos $(68,9 \%)$, por colangiopancreatografia endoscópica retrógrada (ERCP) en 8 casos $(27,5 \%)$ y por colangiografia intraoperatoria en uno $(3,4 \%)$. En los casos de patología maligna el diagnóstico se estableció con biopsia en $27(64,3 \%)$ casos y por imagenología y seguimiento en $15(35,7 \%)$ casos. La mediana de sobrevida de estos últimos fue de 2 meses (rango 1-9 meses). Del total de pacientes con cáncer, 28 $(66,7 \%)$ fueron irresecables. Los diagnósticos y su frecuencia se resumen en la Tabla 1.
Las características epidemiológicas y de laboratorio de los grupos de patología benigna y maligna se resumen en la Tabla 2. Se encontraron diferencias estadísticamente significativas en relación al sexo, valores de CA 19-9, bilirrubina total y fosfatasas alcalinas $(p<0,05)$. No realizamos análisis respecto a los valores de PCR debido a que no todos los pacientes tenían registro de sus valores. Al dicotomizar las variables CA 19-9, bilirrubina total y fosfatasas alcalina; el CA 19-9 >37 U/ml, la bilirrubina total $>10 \mathrm{mg} / \mathrm{dl}$ y las fosfatasas alcalinas $>600 \mathrm{U} / \mathrm{L}$, se asociaron de forma estadísticamente significativa con el diagnóstico de enfermedad maligna $(\mathrm{p}<0,01)$ (Tabla 3).

Los valores de Ca 19-9 fueron mayores en los adenocarcinomas ampulares, tumores periampulares no especificados y cáncer vesicular. El valor más alto de Ca 19-9 se vió en estos últimos, con un valor de $20.000 \mathrm{U} / \mathrm{ml}$. Las medianas y rangos por diagnóstico se resumen en la Tabla 4. Buscamos la existencia de correlación entre los niveles de CA 19-9 con la bilirrubina total, fosfatasas alcalinas y recuento de leucocitos, sin encontrar relación significativa, ni en patología benigna ni maligna (Figuras 1 y 2 ; Tabla 5 ).

Para un nivel de corte de $37 \mathrm{U} / \mathrm{ml}$, la sensibilidad, especificidad, VPP y VPN para diferenciar enfermedad benigna de maligna fue de $81 \%, 72,4 \%, 81 \%$, y $72,4 \%$, respectivamente (Tabla 6). La razón de probabilidad o likelihood ratio (LR) positivo fue de 2,93 y el LR negativo fue de 0,26.

Tabla 1. Resumen de diagnósticos $(n=71)$

\begin{tabular}{|lrr|}
\hline Diagnóstico & n & \% \\
Enfermedad maligna & & \\
Cáncer vesicular & 21 & 29,6 \\
Tumor periampular & 8 & 11,3 \\
Adenocarcinoma ampular & 4 & 5,6 \\
Colangiocarcinoma & 3 & 4,2 \\
Hepatocarcinoma & 3 & 4,2 \\
Adenocarcinoma de páncreas & 3 & 4,2 \\
Enfermedad benigna & & \\
Colecistitis crónica & 9 & 12,7 \\
Colecistitis crónica + CLC & 6 & 8,5 \\
Colecistitis aguda & 2 & 2,8 \\
Colecistitis aguda + CLC & 3 & 4,2 \\
Coledocolitiasis & 9 & 12,7 \\
Total & 71 & 100 \\
\hline
\end{tabular}


Tabla 2. Características epidemiológicas y de laboratorio de los grupos con patología benigna y maligna

\begin{tabular}{|c|c|c|c|}
\hline & Benigno $(n=29)$ & Maligno $(n=42)$ & $\mathbf{p}$ \\
\hline \multicolumn{4}{|l|}{ Edad (años) } \\
\hline Media (ds) & $59,9(19,8)$ & $65,3(11,3)$ & 0,07 \\
\hline Mediana (rango) & $62(20-88)$ & $67,0(40-85)$ & --- \\
\hline \multicolumn{4}{|l|}{ Sexo femenino } \\
\hline $\mathrm{n}$ & 26 & 28 & \\
\hline$\%$ & 89,7 & 66,7 & 0,013 \\
\hline \multicolumn{4}{|l|}{ CA $19-9(\mathrm{U} / \mathrm{ml})$} \\
\hline Media (ds) & $43,7(83,9)$ & $1.160,7(3.245,6)$ & --- \\
\hline Mediana (rango) & $15(0-431,1)$ & $220,8(0-20.000)$ & $<0,0001$ \\
\hline \multicolumn{4}{|c|}{ Bilirrubina total (mg/dl) } \\
\hline Media (ds) & $4,1(3,8)$ & $7,5(5,7)$ & 0,003 \\
\hline Mediana (rango) & $3,5(0,2-13,7)$ & $7,9(0,1-18,6)$ & ---- \\
\hline \multicolumn{4}{|c|}{ Fosfatasas alcalinas (UI/I) } \\
\hline Media (ds) & $243,4(165,9)$ & $654,2(574,7)$ & -- \\
\hline Mediana (rango) & $199(21-633)$ & $554,5(3,6-3.201)$ & $<0,0001$ \\
\hline \multicolumn{4}{|c|}{ Leucocitos $\left(/ \mathrm{mm}^{3}\right)$} \\
\hline Media (ds) & $9.832(3.439)$ & $11.736,2(5.671,5)$ & --- \\
\hline Mediana (rango) & $9.500(2.770-17.700)$ & $10.600(5.600-31.00)$ & 0,13 \\
\hline
\end{tabular}

Tabla 3. Análisis de las variables CA 19-9, bilirrubina total y fosfatasas alcalinas, dicotomizadas y su asociación con el diagnóstico de enfermedad maligna

\begin{tabular}{|c|c|c|c|c|c|}
\hline \multirow[t]{2}{*}{ Variable } & \multicolumn{2}{|c|}{ Maligna $(n=42)$} & \multicolumn{2}{|c|}{ Benigna $(n=29)$} & \multirow[t]{2}{*}{$\mathbf{p}$} \\
\hline & $\mathbf{n}$ & $\%$ & n & $\%$ & \\
\hline \multicolumn{6}{|l|}{ CA 19-9 } \\
\hline$>37 \mathrm{U} / \mathrm{ml}$ & 34 & 81 & 8 & 27,6 & \multirow[t]{2}{*}{$<0,0001$} \\
\hline$\leq 37 \mathrm{U} / \mathrm{ml}$ & 8 & 19 & 21 & 72,4 & \\
\hline \multicolumn{6}{|c|}{ Bilirrubina total } \\
\hline$>10 \mathrm{mg} / \mathrm{dl}$ & 16 & 38,1 & 2 & 6,9 & \multirow[t]{2}{*}{0,003} \\
\hline$\leq 10 \mathrm{mg} / \mathrm{dl}$ & 26 & 61,9 & 27 & 93,1 & \\
\hline \multicolumn{6}{|c|}{ Fosfatasas alcalinas } \\
\hline$>600 \mathrm{UI} / \mathrm{L}$ & 18 & 42,9 & 1 & 3,4 & \multirow[t]{2}{*}{0,0002} \\
\hline$\leq 600 \mathrm{UI} / \mathrm{L}$ & 24 & 57,1 & 28 & 96,6 & \\
\hline
\end{tabular}

Tabla 4. Medianas y rangos de los niveles de CA 19-9 en relación al diagnóstico y proporción de casos con niveles de CA 19-9 > 37 U/ml

\begin{tabular}{|c|c|c|c|c|c|}
\hline \multirow[t]{2}{*}{ Diagnóstico } & \multirow[t]{2}{*}{$\mathbf{n}$} & \multicolumn{2}{|c|}{ Ca $19-9(\mathrm{U} / \mathrm{ml})$} & \multicolumn{2}{|c|}{ Ca $19-9>37(\mathrm{U} / \mathrm{ml})$} \\
\hline & & Mediana & Rango & $\mathbf{N}$ & $\%$ \\
\hline Cáncer vesicular & 21 & 221,1 & $(0-20000)$ & 18 & 85,7 \\
\hline Tumores periampulares & 8 & 328,3 & $(0-5000)$ & 7 & 87,5 \\
\hline Colangiocarcinoma & 3 & 0,4 & $(0-742,5)$ & 1 & 33,3 \\
\hline Hepatocarcinoma & 3 & 220,4 & $(12,4-1286)$ & 2 & 66,7 \\
\hline Adenocarcinoma ampular & 4 & 350,4 & $(67,3-569,3)$ & 4 & 100,0 \\
\hline Adenocarcinoma de páncreas & 3 & 75,2 & $(0-443)$ & 2 & 66,7 \\
\hline Enfermedad maligna & 42 & 220,8 & $(0-20000)$ & 34 & 81,0 \\
\hline Enfermedad biliar litiásica & 29 & 15,0 & $(0-431)$ & 8 & 27,5 \\
\hline
\end{tabular}




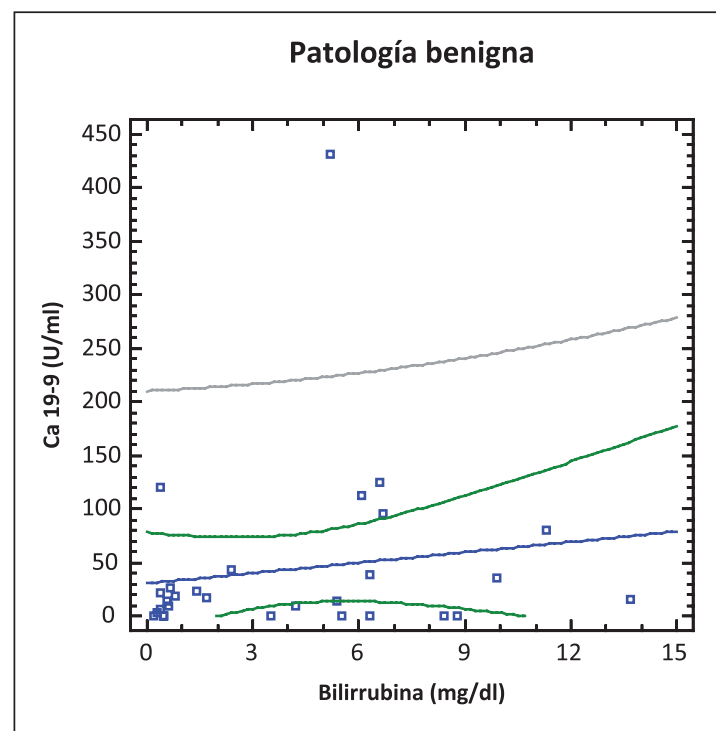

Figura 1. Regresión lineal entre los valores de CA 19-9 $\mathrm{y}$ de bilirrubina total en patología benigna $(\mathrm{n}=29)$; $(\mathrm{r}=0,14$ y $\mathrm{p}=0,44)$.

Tabla 5. Análisis de regresión lineal entre los valores de CA 19-9 con los valores de bilirrubina total, fosfatasas alcalinas y recuento de leucocitos en patología benigna $(n=29)$ y maligna $(n=42)$

\begin{tabular}{|lccccc|}
\hline \multirow{2}{*}{ Parámetro } & \multicolumn{4}{c|}{ CA 19-9 (U/ml) } \\
& Benigno & \multicolumn{3}{c|}{ Maligno } \\
& r & $\mathbf{p}^{*}$ & $\mathbf{r}$ & $\mathbf{p}^{*}$ \\
Bilirrubina total $(\mathrm{mg} / \mathrm{dl})$ & 0,14 & 0,44 & 0,26 & 0,09 \\
Fosfatasas alcalinas $(\mathrm{U} / \mathrm{L})$ & 0,20 & 0,28 & 0,08 & 0,6 \\
Recuento de leucocitos $\left(\mathrm{mm}^{3}\right)$ & 0,22 & 0,23 & 0,14 & 0,34 \\
\hline
\end{tabular}

r: Coeficiente de correlación.

*: Valor p calculado en base a la Tabla ANOVA.

Tabla 6. Tabla de contingencia con los resultados de CA 19-9 y diagnóstico definitivo para un nivel de corte de $37 \mathrm{U} / \mathrm{ml}$

\begin{tabular}{|c|c|c|c|c|}
\hline & & \multicolumn{2}{|c|}{ Diagnóstico definitivo } & \multirow[b]{2}{*}{ Total } \\
\hline & & Maligno & Benigno & \\
\hline \multirow{3}{*}{$\begin{array}{l}\text { CA } 19-9 \\
>37 \mathrm{U} / \mathrm{ml}\end{array}$} & $(+)$ & 34 & 8 & 42 \\
\hline & $(-)$ & 8 & 21 & 29 \\
\hline & Total & 42 & 29 & 71 \\
\hline
\end{tabular}

Sensibilidad $=81 \%$, Especificidad $=72,4 \%$, VPP $=81 \%$ y $\mathrm{VPN}=72,4 \%$.

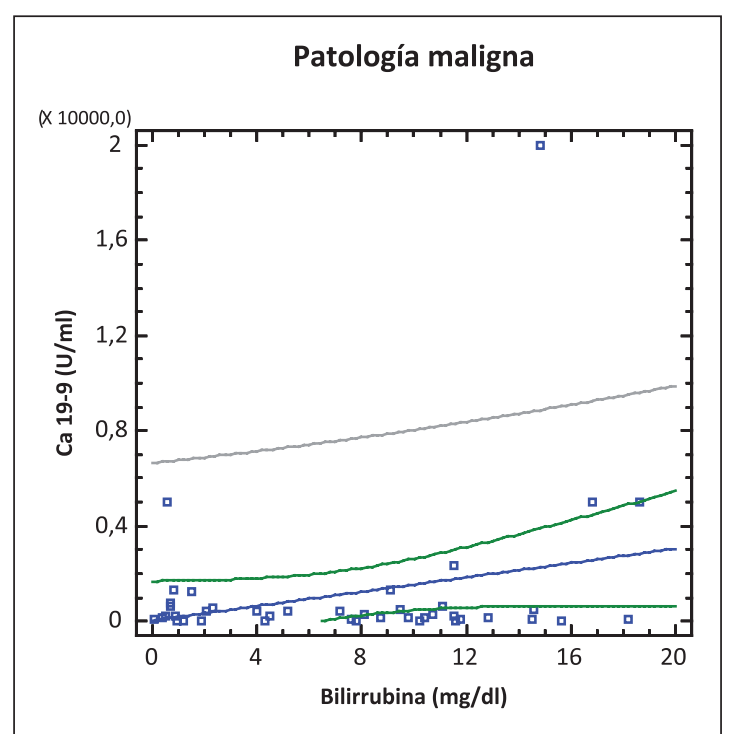

Figura 2. Regresión lineal entre los valores de CA 19-9 y de bilirrubina total en patología maligna $(n=42)$; $(\mathrm{r}=0,26$ у $\mathrm{p}=0,09)$.

La construcción de la Curva ROC arrojó un área bajo la curva de 0,816 (95\% CI: 0,715-0,917; Figura 3). Esto permitió identificar un valor óptimo de $\mathrm{Ca}$ 19-9 de $130 \mathrm{U} / \mathrm{ml}$, para el cual la sensibilidad, especificidad, VPP y VPN para diferenciar enfermedad benigna de maligna fueron de $64,2 \%, 96,6 \%, 96,4 \%$ y $65,1 \%$, respectivamente (Tabla 7 ). El LR positivo fue de 18,6 y el LR negativo fue de 0,36 .

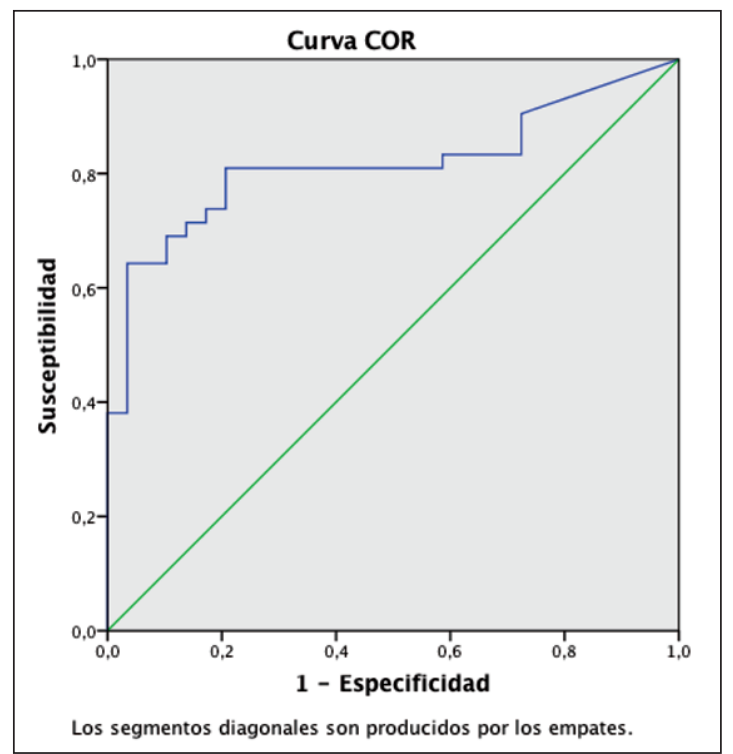

Figura 3. Curva ROC para CA 19-9. Área bajo la curva de 0,816 (95\% CI: 0,715-0,917). 
Tabla 7. Valores de sensibilidad, especificidad, VPP y VPN (\%) para distintos valores de corte de CA 19-9 (U/ml)

\begin{tabular}{|ccccc|}
\hline CA 19-9 (U/ml). & Sensibilidad & Especificidad & VPP & VPN \\
$>37$ & 81,0 & 72,4 & 81,0 & 72,4 \\
$>70$ & 76,1 & 79,3 & 84,2 & 69,7 \\
$>100$ & 71,4 & 86,2 & 88,2 & 67,6 \\
$>130$ & 64,3 & 96,6 & 96,4 & 65,1 \\
\hline
\end{tabular}

\section{Discusión}

El CA 19-9 es una glicoproteína expresada por varios cánceres epiteliales así como también en células epiteliales normales del páncreas, conductos biliares, saliva y meconio ${ }^{13}$. Desde que se inicio su uso en la práctica clínica no se ha logrado establecer adecuadamente su rol en el manejo de los pacientes oncológicos, principalmente debido al alto número de falsos positivos ${ }^{14}$. Además, un resultado negativo no excluye el diagnóstico de malignidad dado que el $5 \%$ de la población y por ende un $5 \%$ de los carcinomas podrían no expresar el marcador ${ }^{15}$.

En cáncer de páncreas se han reportado sensibilidad y especificidad diagnósticas de $80-90 \%$ y $70-80 \%$, respectivamente ${ }^{14,16}$, y en tumores de la vía biliar las cifras reportadas son de $68-77 \%$ y $75-85 \%$, respectivamente ${ }^{6,17}$.

En nuestro estudio intentamos evaluar la capacidad del CA 19-9 para distinguir patología biliopancreática benigna y maligna. Encontramos que los valores de CA 19-9 fueron mayores en el grupo neoplásico, lo que fue estadísticamente significativo (Tabla 2). Las neoplasias con las medianas más elevadas fueron los adenocarcinomas ampulares y los tumores periampulares no especificados, pero el valor más alto lo encontramos en un cáncer vesicular con $20.000 \mathrm{U} / \mathrm{ml}$ (Tabla 4). Bajo $440 \mathrm{U} / \mathrm{ml} \mathrm{la}$ distribución de enfermedades malignas y benignas fue dividida (27 y 29 casos respectivamente), sin embargo, sobre $440 \mathrm{U} / \mathrm{ml}$ todos los pacientes tuvieron diagnóstico de malignidad.

Dada la presencia casi constante de hiperbilirrubinemia en los pacientes estudiados con CA 19-9 por patología bilio-pancreática, varios estudios han evaluado su relación. Mann y cols ${ }^{9}$, analizaron un grupo de 164 pacientes con CA 19-9 elevado sobre $33 \mathrm{U} / \mathrm{ml}$ asociado a hiperbilirrubinemia, encontrando una correlación positiva en el subgrupo de pacientes con patología benigna $(r=0,41$ y $\mathrm{p}<0,01)$, no así en el subgrupo de pacientes con patología maligna. Ong y cols ${ }^{18}$, en 83 pacientes con patología biliar benigna y CA 19-9 y bilirrubina total elevada, también encontraron correlación positiva $(\mathrm{r}=0,363$ y $\mathrm{p}<0,001)$ y Dogan y cols ${ }^{19}$, en 32 pacientes con coledocolitiasis con y sin colangitis aguda también encontraron una correlación positiva con los valores de bilirrubina $(r=0,4$ y $p<0,001)$ y fosfatasas alcalinas $(r=0,5$ y $p<0,01)$. En nuestra serie no encontramos correlación entre los valores de CA 19-9 y bilirrubina, así como tampoco con los niveles de fosfatasas alcalinas, en los pacientes con enfermedad benigna ni maligna (Figura 1 y 2; Tabla 5), lo que podría estar influenciado por el bajo número de pacientes.

Algunas de las enfermedades benignas que forman parte de los falsos positivos del CA 19-9, son patologías inflamatorias, por lo que buscamos la relación entre el CA 19-9 y el recuento de leucocitosis (como marcador inflamatorio), sin encontrar correlación significativa (Tabla 5). Debido a que no todos los pacientes tenían registro de la PCR, no pudimos analizar su correlación con el CA 19-9.

En la evaluación del CA 19-9 como examen capaz de diferenciar patología bilio-pancreática benigna y maligna, la mayoría de la literatura indica que el límite de la normalidad para el CA 19-9 $(<37$ U/ $\mathrm{ml})$, tiene un bajo rendimiento, y varios reportes han buscado cambiar el nivel de referencia. Morris-Stiff G. y cols ${ }^{11}$, encontraron una sensibilidad, especificidad, VPP y VPN de $84,9 \%, 69,7 \%, 67,7 \%$ y $86,1 \%$, respectivamente. Al subir el valor de corte a 70,5 $\mathrm{U} / \mathrm{ml}$, mediante la construcción de una curva ROC, la sensibilidad bajó a $82,1 \%$, pero la especificidad, VPP y VPN mejoraron a $85,9 \%, 81,3 \%$ y $86,5 \%$, respectivamente. Marelli y cols ${ }^{12}$, en pacientes con ictericia obstructiva encontraron una sensibilidad de $86 \%$ y una especificidad de $39 \%$. Al subir el nivel de corte a $90 \mathrm{U} / \mathrm{ml}$, mediante la construcción de una curva ROC, la sensibilidad y especificidad fueron de $75 \%$ y $61 \%$, respectivamente.

En nuestro estudio para un valor de corte de 37 $\mathrm{U} / \mathrm{ml}$, la sensibilidad, especificidad, VPP y VPN del CA 19-9 para diferenciar enfermedad benigna de maligna fue de $81 \%, 72,4 \%, 81 \%$ y $72,4 \%$, respectivamente (Tabla 6). La prevalencia de enfermedad maligna en la serie fue de $59,2 \%$ (probabilidad pre test), con un LR positivo de 2,93, la probabilidad post test fue de $78 \%$, y con un LR negativo de 0,26 ; la probalidad post test fue de $28 \%$, lo que 
demuestra el bajo rendimiento de la prueba como test diagnóstico.

A través de la construcción de una curva ROC buscamos mejorar el rendimiento del examen. A medida que aumentamos el valor de corte, fuimos logrando una mejoría en la especificidad y VPP, lo que significó una disminución de la sensibilidad y el VPN (Tabla 7). Con un nivel de corte de 130 $\mathrm{U} / \mathrm{ml}$ logramos una especificidad y VPP de $96,6 \%$ y $96,4 \%$, respectivamente. La probalidad post test con un LR positivo de 18,6 aumentó a $95 \%$ y con un LR negativo de 0,36 fue de $30 \%$ (similar al observado con el valor de normalidad). Observamos así, que este nuevo valor de corte encontrado mejora el rendimiento de la prueba, resultando en un buen predictor de malignidad.

Nuestros resultados muestran que el marcador tumoral del CA 19-9 es útil en el proceso diagnóstico de patología bilio-pancreática benigna y maligna, con un valor de corte sobre el límite de la normalidad. Su utilidad principal estaría en la categorización de pacientes con enfermedad bilio-pancreática para guiar y priorizar los estudios en aquellos con sospecha de enfermedad maligna, así como preparar al equipo tratante a un enfrentamiento quirúrgico con criterio oncológico.

El marcador CA 19-9 no debe ser utilizado en forma individual, pero sí como un apoyo a la historia clínica y a la sospecha de patología maligna. Su uso asociado a estudios imagenológicos permite mejorar en forma importante su rendimiento diagnóstico ${ }^{11}$, sin embargo, el aporte real del marcador a las imágenes es desconocido. Por otra parte, si bien se ha reportado una relación entre los valores de CA 19-9 y los valores de bilirrubina total en enfermedad benigna, nuestros resultados son incapaces de apoyar esa hipótesis.

Una limitación importante de nuestro estudio es el número de pacientes. Sin embargo, se incluyó un espectro apropiado con enfermedades distintas pero parecidas en su presentación clínica. Es importante el hecho de que el $66,7 \%$ de los pacientes con cáncer fueron irresecables. Sin embargo, al excluir a este grupo, las medianas de CA 19-9 de los pacientes con patología benigna siguieron siendo significativamente menores que la de los pacientes con patología maligna resecable $(15 \mathrm{U} / \mathrm{ml}(0-431,1)$ vs $432,4 \mathrm{U} / \mathrm{ml}$ $(0-1.299,1) ; \mathrm{p}<0,001)$.

Otro punto importante es que si bien no todos los pacientes tuvieron el mismo gold estándar, debido a los diferentes diagnósticos, estos son aceptados por la comunidad médica (la colangiografía para la coledocolitiasis y las imágenes y seguimiento en pacientes con cáncer no susceptibles de procedimientos biópticos) como lo sugieren guías de estudios de pruebas diagnósticas ${ }^{20,21}$.

\section{Conclusiones}

Los valores de CA 19-9 son significativamente mayores en patología maligna. No encontramos una relación entre los valores de bilirrubina, fosfatasas alcalinas, recuento de leucocitos y el Ca 19-9, ni en patología benigna ni maligna. La utilidad diagnóstica del marcador con un valor de corte de $37 \mathrm{U} / \mathrm{ml}$ es baja, sin embargo, la optimización de éste a un valor de $130 \mathrm{U} / \mathrm{ml}$, ayuda a mejorar su capacidad de predecir malignidad. Nuestros resultados deben valorarse con cautela, y servir de base para futuros estudios prospectivos con respecto a este tema.

\section{Referencias}

1. Sokoll L, Chan D. Bioquímica clínica: estudios de marcadores tumorales. En: Abeloff M, ed. Oncología clínica. España: Editorial Elsevier España, 2005;329-33.

2. Karachristos A, Scarmeas N, Hoffman JP. CA 19-9 levels predict results of staging laparoscopy in pancreatic cancer. J Gastrointest Surg. 2005;9:1286-92.

3. Ferrone CR, Finkelstein DM, Thayer SP, Muzikansky A, Fernández-del Castillo C, Warshaw AL. Perioperative CA19-9 levels can predict stage and survival in patients with resectable pancreatic adenocarcinoma. J Clin Oncol. 2006;24:2897-902.

4. Goonetilleke KS, Siriwardena AK. Systematic review of carbohydrate antigen (CA 19-9) as a biochemical marker in the diagnosis of pancreatic cancer. Eur J Surg Oncol. 2007;33:266-70.

5. Patel AH, Harnois DM, Klee GG, LaRusso NF, Gores GJ. The utility of CA 19-9 in the diagnoses of cholangiocarcinoma in patients without primary sclerosing cholangitis. Am J Gastroenterol. 2000;95:204-7.

6. Qin XL, Wang ZR, Shi JS, Lu M, Wang L, He QR. Utility of serum CA19-9 in diagnosis of cholangiocarcinoma: in comparison with CEA. World J Gastroenterol. 2004; 10:427-32.

7. Lukaszewicz-Zając M, Mroczko B, Gryko M, Kędra B, Szmitkowski M. Comparison between clinical significance of serum proinflammatory proteins (IL-6 and CRP) and classic tumor markers (CEA and CA 19-9) in gastric cancer. Clin Exp Med. 2011;11:89-96.

8. Sato H, Usuda N, Kuroda M, Hashimoto S, Maruta M, Maeda K. Significance of serum concentrations of E-selectin and CA 19-9 in the prognosis of colorectal Cancer. Jpn J Clin Oncol. 2010;40:1073-80.

9. Mann DV, Edwards R, Ho S, Lau WY, Glazer G. Elevated tumour marker CA19-9: clinical interpretation and influence of obstructive jaundice. Eur J Surg Oncol. 2000;26:474-9.

10. Sheen-Chen SM, Sun CK, Liu YW, Eng HL, Ko SF, Kuo CH. Extremely elevated CA19-9 in acute cholangitis. Dig Dis Sci. 2007;52:3140-2.

11. Morris-Stiff G, Teli M, Jardine N, Puntis MC. CA19- 
9 antigen levels can distinguish between benign and malignant pancreaticobiliary disease. Hepatobiliary Pancreat Dis Int. 2009;8:620-6.

12. Marrelli D, Caruso S, Pedrazzani C, Neri A, Fernandes E, Marini M, et al. CA19-9 serum levels in obstructive jaundice: clinical value in benign and malignant conditions. Am J Surg. 2009;198:333-9.

13. Koprowski H, Steplewski Z, Mitchell K, Herlyn M, Herlyn D, Fuhrer P. Colorectal carcinoma antigens detected by hybridoma antibodies. Somat Cell Genet. 1979;5:957-71.

14. Goonetilleke KS, Siriwardena AK. Systematic review of carbohydrate antigen (CA 19-9) as a biochemical marker in the diagnosis of pancreatic cancer. Eur J Surg Oncol. 2007;33:266-70.

15. Tempero MA, Uchida E, Takasaki H, Burnett DA, Steplewski Z, Pour PM. Relationship of carbohydrate antigen 19-9 and Lewis antigens in pancreatic cancer. Cancer Res. 1987;47:5501-3.

16. Kim HJ, Kim MH, Myung SJ, Lim BC, Park ET, Yoo
KS, et al. A new strategy for the application of CA19-9 in the differentiation of pancreaticobiliary cancer: analysis using a receiver operating characteristic curve. Am J Gastroenterol. 1999;94:1941-6.

17. Li YG, Zhang N. Clinical significance of serum tumour M2-PK and CA19-9 detection in the diagnosis of cholangiocarcinoma. Dig Liver Dis. 2009;41:605-8.

18. Ong SL, Sachdeva A, Garcea G, Gravante G, Metcalfe MS, Lloyd DM, et al. Elevation of carbohydrate antigen 19.9 in benign hepatobiliary conditions and its correlation with serum bilirubin concentration. Dig Dis Sci. 2008;53:3213-7.

19. Doğan ÜB, Gümürdülü Y, Gölge N, Kara B. Relationship of CA 19-9 with choledocholithiasis and cholangitis. Turk J Gastroenterol. 2011;22:171-7.

20. Burgos M, Manterola C. Cómo interpretar un artículo sobre pruebas diagnósticas. Rev Chil Cir. 2010;62:3018.

21. Valenzuela L, Cifuentes L. Validez de estudios de tests diagnósticos. Rev Med Chile 2008;136:401-4. 